\title{
Editorial
}

\section{AI Powered Service Optimization for Edge/Fog Computing}

\author{
Sang-Bing Tsai $\mathbb{D}^{1},{ }^{1}$ Chia-Hui Wu $\mathbb{D}^{2},{ }^{2}$ Xiaolong $X u,{ }^{3}$ Yuan Yuan, ${ }^{4}$ and Qiang $\mathrm{He}^{5}$ \\ ${ }^{1}$ Regional Green Economy Development Research Center, School of Business, WUYI University, Nanping, China \\ ${ }^{2}$ Department of Hotel Management and Culinary Creativity, Minghsin University of Science and Technology, Xinfeng, Taiwan \\ ${ }^{3}$ Nanjing University of Information Science and Technology, Nanjing, China \\ ${ }^{4}$ Michigan State University, East Lansing, MICH, USA \\ ${ }^{5}$ Swinburne University of Technology, Melbourne, Australia
}

Correspondence should be addressed to Chia-Hui Wu; chiahuei530@gmail.com

Received 30 November 2021; Accepted 30 November 2021; Published 11 December 2021

Copyright (c) 2021 Sang-Bing Tsai et al. This is an open access article distributed under the Creative Commons Attribution License, which permits unrestricted use, distribution, and reproduction in any medium, provided the original work is properly cited.

In recent years, the explosive development of Internet of Things (IoT) has generated a large amount of data from both the user-device side and the network side, which challenges the traditional cloud-based data transmission, storage, and processing applications in terms of efficiency, security, and economic cost. In this situation, the newly emerged computing paradigms, Edge Computing and Fog Computing, have complemented the traditional cloud-based systems. Edge/Fog can provide partial computing resources closer to the user or device side; certain computing tasks can thereby be executed or processed directly by the close Edge/Fog resources without sending them to the distant cloud centre. This can reduce the load of a cloud platform, and the task execution efficiency can be improved significantly.

However, existing Edge/Fog-based service systems often suffer from limited computing capabilities, high energy cost, and fast changing context environment, which call for intelligent optimization of business strategies adopted in both the user-device side and network side. Fortunately, artificial intelligence (AI) technology provides a promising way to achieve the above Edge/Fog service optimization goals. However, the integration of AI and Edge/Fog techniques is still a challenging issue that needs intensive study. In view of the above analyses, this special issue aims to highlight the cutting-edge research and applications related to the "AI Powered Service Optimization for Edge/Fog Computing."

In this special issue, we look for significant findings in tackling new security issues that challenge artificial intelligence in the mobile edge computing environment. Specifically, we solicit novel contributions on secure artificial intelligence from a variety of perspectives, e.g., architecture, data, and algorithms. This special issue has collected some good articles. It had great repercussions and success.

\section{Conflicts of Interest}

The guest editors declare no conflicts of interest.

\section{Acknowledgments}

We thank all authors for their participation.

Sang-Bing Tsai Chia-Hui Wu Xiaolong $X u$ Yuan Yuan Qiang He 